\title{
Two Cases of Ascending Aortic Intramural Hematoma Combined with Cardiac Tamponade Treated with Different Strategies
}

\author{
Min-Suk Kim ', Tae-Jin Kim ', Jong Joo Moon', Jae-Hyung Nam ', Dong-Hoon Han', Ji-Hun Ahn', Tae-Hoon Kim², Do-Hoi Kim', \\ Young-Nam Youn ${ }^{3}$, Yoon-Nyun Kim ${ }^{4}$ \\ 'Division of Cardiology, Soonchunhyang University Gumi Hospital, Soonchunhyang University College of Medicine, Gumi; '²ivision of Cardiology, Chungdam \\ Wooridul Hospital; ${ }^{3}$ Division of Cardiovascular Surgery, Yonsei Cardiovascular Hospital, Yonsei University College of Medicine, Seoul; ${ }^{4}$ Division of Cardiology, \\ Keimyung University Dongsan Medical Center, Keimyung University School of Medicine, Daegu, Korea
}

Aortic intramural hematoma (IMH) is classically defined as a variation of aortic dissection where blood collects within the aortic media without the presence of an intimal flap. Ascending IMH is known to have worse clinical outcomes than IMH of the descending aorta or aortic arch. Therefore, some patients with higher risk of disease progression require surgical corrections. However, the indications and the benefits of surgical management of ascending IMH, compared with medical treatment only, have not yet been established. We present two cases of IMH in the ascending aorta that presented with cardiac tamponade; patients were treated differently according to risk factors.

Keywords: Intramural hematoma; Cardiac tamponade; Penetrating ulcer

\section{INTRODUCTION}

Cardiac tamponade frequently complicates acute proximal aortic dissection and is known as one of the most common causes of death. Having similar pathophysiology, cardiac tamponade can also occur in patients with intramural hematoma (IMH) [1]. It results from blood leaking from the false lumen to the pericardial space during aortic dissection [2]. Therefore, in prompt response to the lethal complication, pericardiocentesis is indicated as the first choice of treatment in patients with cardiac tamponade to stabilize patient hemodynamics [2]. Although open aortic surgery is usually considered due to the risk of progression to classic aortic dissection or aortic rupture, it is not compulsory for all patients who achieve hemodynamic stability [3,4]. A recent study revealed that symptomatic patients with penetrating ulcers of the ascending aorta might have worse prognosis requiring an urgent surgical repair of what is considered a distinct pathologic variant of classic aortic dissection. Penetrating aortic ulcer describes a condition in which an atherosclerotic plaque tears and ulcerates through the media, leading to a variable amount of intramural hematoma formation [5]. However, despite the importance of this finding, the exact pathology has not been well defined. We present here two patients with ascending IMH combined with cardiac tamponade. By comparing radiologic images with true pathology, our cases provide insight into penetrating ulcers; also, the case of the patient without risk factors illustrates good long-term (up to 10 years) outcome.

\section{CASE REPORTS}

\section{Case 1}

A 75-year-old woman with hypertension on medication admitted to our hospital for a New York Heart Association functional class-2 dyspnea. She had felt dyspnea for 1 week but she didn't have any chest pain, radiating pain or fever. Her blood pressure was 100/ $65 \mathrm{mmHg}$, body temperature was $36.5^{\circ} \mathrm{C}$, and she was tachycardic (105 bpm). Chest radiography showed cardiomegaly with pleural effusion in the right lower lung field (Fig. 1A). All the chemistry
Correspondence to: Tae-Hoon Kim

Division of Cardiology, Chungdam Wooridul Hospital, 445 Hakdong-ro, Gangnam-gu, Seoul 135-951, Korea

Tel: +82-2-513-8880, Fax: +82-2-513-8883, E-mail: sch.kimtaehoon@gmail.com

Received: Mar. 9, 2012 / Accepted after revision: Jun. 14, 2012
(C) 2012 Soonchunhyang Medical Research Institute This is an Open Access article distributed under the terms of the Creative Commons Attribution Non-Commercial License (http://creativecommons.org/licenses/by-nc/3.0/). 

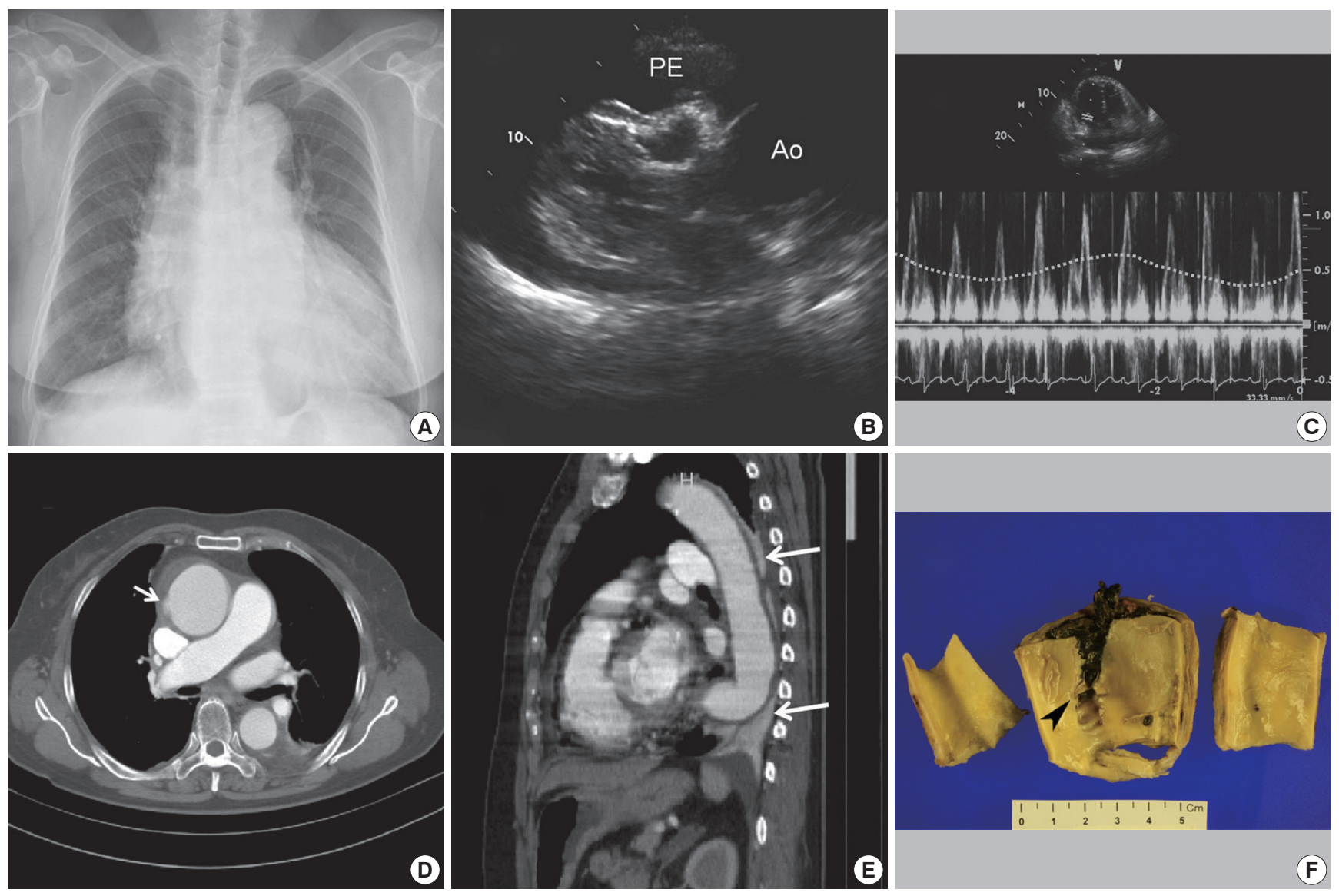

Fig. 1. (A) Chest posteroanterior shows cardiomegaly with right pleural effusion. (B) Transthoracic echocardiography shows the enlarged ascending aorta (Ao) with early diastolic collapse of the right ventricle. (C) Doppler mitral inflow indicates significant respiratory variation (dotted line). (D) An axial contrast enhanced computed tomography (CT) shows intramural hematoma of the ascending and descending aorta. Arrow indicates a focal irregular outpouching as a penetrating ulcer. (E) Reconstructed sagittal cross-section CT indicating high density hemorrhagic leakage through the descending aorta and the pleural space. Arrows indicate the leaked fluid. (F) Gross pathology showed the intimal tear in the ascending aorta (arrowhead). PE, pericardial effusion.

laboratory values were within normal range (protein/albumin, 6.9/3.8 g/dL; total cholesterol, $158 \mathrm{mg} / \mathrm{dL}$; triglyceride, $64 \mathrm{mg} / \mathrm{dL}$; low density lipoprotein-cholesterol, $116 \mathrm{mg} / \mathrm{dL}$ ). However, her hemoglobin level was decreased to $10.4 \mathrm{~g} / \mathrm{dL}$ and brain natriuretic peptide was elevated to $103.5 \mathrm{pg} / \mathrm{mL}$. Transthoracic echocardiography (TTE) was immediately performed after her admission and revealed a large amount of pericardial effusion with swinging heart. The right atrium (RA) was not visualized from the parasternal view due to the enlarged ascending aorta $(47 \mathrm{~mm})$, however the right ventricle (RV) was seen collapsed until early diastole (Fig. 1B). Although it was not fully estimated because of tachycardia, $\mathrm{E}$ mitral flow was a significant variable of respiratory change (Fig. 1C). Therefore, we performed pericardiocentesis on the patient via apical approach. After 1.1 L of drainage, intra-pericardial pressure decreased to a normal level (from 13 to $5 \mathrm{mmHg}$ ) and heart rate re- duced to $80 \mathrm{bpm}$. Over 100,000 red blood cells were counted with $60 \%$ in fresh form in pericardial fluid while fluid protein and albumin levels were similar to serum levels (protein $6.2 \mathrm{~g} / \mathrm{dL}$ and albu$\min 3.5 \mathrm{~g} / \mathrm{dL}$ in pericardial fluid versus protein $6.9 \mathrm{~g} / \mathrm{dL}$ and albu$\min 3.8 \mathrm{~g} / \mathrm{dL}$ in serum). Computed tomography (CT) was performed to evaluate the cause of hemopericardium. The axial contrast image ascending through the descending thoracic aorta revealed a crescentic, high attenuation material within the aortic wall, consistent with intramural hematoma. Also a focal bulge out of the ascending aorta extending beyond the intimal border was seen in the axial view (Fig. 1D). A high density fluid collection was visualized through the descending aorta and the dependent portion of the pleural space (Fig. 1E). Although the patient did not complain of dyspnea after pericardial drainage, we decided to treat this patient with an open surgery. Epicardium was adhered to the 

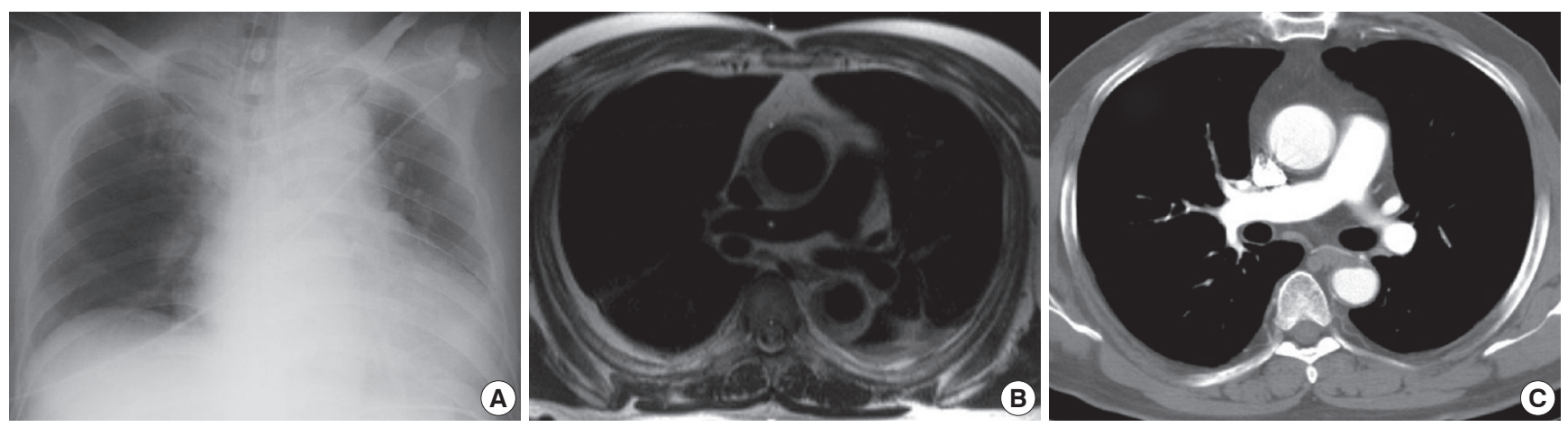

Fig. 2. (A) Chest radiography shows widened mediastinum and cardiomegaly. (B) An axial magnetic resonance imaging shows intramural hematoma of the ascending and descending aorta without tear sites. (C) A 10-year follow-up computed tomography shows no visible crescentic, high attenuation material within the aortic wall at the ascending or descending aorta.

myocardium and the ascending aorta was also adhered to the RA. There was an intimal tear site on the distal part of the ascending aorta (Fig. 1F). During 6-month clinical follow-up after aorta graft replacement surgery, she showed excellent functional capacity without any sequelae.

\section{Case 2}

A 52-year-old man with hypertension on medication presented to the emergency department with acute chest pain. His blood pressure was $80 / 60 \mathrm{mmHg}$ and heart rate was $110 \mathrm{bpm}$. Chest radiography showed a widened mediastinum and cardiomegaly (Fig. 2A). The CT taken at the primary hospital revealed ascending IMH with enhanced pericardial effusion. The emergency portable TTE revealed the collapse of RA with early diastolic collapse of RV and a large amount of pericardial effusion. After immediate pericardiocentesis, the patient's vital signs stabilized. Ten days later, magnetic resonance imaging showed neither the visible intimal tear site nor the residual pericardial hemorrhage (Fig. 2B). The patient was discharged on medications including atenolol. During ten years of follow-up, the patient has not had any chest pain. Also the 10-year follow-up CT showed no visible false lumen around the ascending or descending aorta (Fig. 2C).

\section{DISCUSSION}

$\mathrm{IMH}$, defined as aortic dissection without identifiable intimal tears and lack of flow in the aorta false lumen [6], was known to have similar natural history and prognosis to classic dissection and therefore was classified according to location of involvement of the ascending or descending aorta [7]. Generally, there is con- sensus about treatment of patients with uncomplicated IMH involving only the descending aorta or the aortic arch; optimal medical treatment is recommended for these patients [6]. However, until recently, the optimal treatment of ascending IMH remained controversial. IMH is believed to have a more benign natural history than classic aortic dissection. However, patients with IMH showed a greater frequency of pericardial and pleural effusion, with a higher prevalence of cardiac tamponade at clinical presentation [8]. Although the prevalence of cardiac tamponade was higher than that in classic aortic dissection, the IMH patients presenting with tamponade and surviving after the pericardiocentesis turned out to have their conditions improved during medical therapy $[3,4]$. Therefore, physicians must decide on further treatment strategies following pericardial drainage.

Currently, the most important markers requiring open surgery in IMH patients seemed to be the patient's ongoing chest pain and existence of penetrating ulcers of the ascending aorta [5]. Penetrating aortic ulcer is defined by an ulceration of an aortic atherosclerotic plaque penetrating through the internal elastic lamina into the aortic media [9]. The penetrating ulcer could separate media layers and could lead to the adjacent IMH to either a local or longitudinal progression. Our pathology assessment also demonstrated longitudinal tearing at the ascending aorta which made a true opening through the media (Fig. 1F). For this reason, it was known as a denominator of IMH progression and an adverse outcome [7]. Many authors believed that penetrating ulcers located at the ascending aorta probably require urgent surgical repair in most patients [10, 11]. Proximally located IMH in patients aged over 55 with over 56 $\mathrm{mm}$ of local aortic diameter was a predictor of IMH progression in a multivariate analysis [7]. Despite the patient in case 1 not com- 
plaining of ongoing pain, we decided treatment with aggressive open surgery because of the advanced patient's age and the location of the intramural hematoma at ascending aorta, while we have medically treated the patient in case 2 , and he has survived 10 years without complications.

Both cases presented with cardiac tamponade resulting from hemopericardium due to an ascending IMH. Although hemodynamic stabilization was achieved after pericardiocentesis in both cases, due to the higher risks of overt dissection or rupture, one patient underwent aortic surgery and successfully survived the crisis.

\section{REFERENCES}

1. Estrera A, Miller C 3rd, Lee TY, De Rango P, Abdullah S, Walkes JC, et al. Acute type A intramural hematoma: analysis of current management strategy. Circulation 2009;120(11 Suppl):S287-91.

2. Isselbacher EM, Cigarroa JE, Eagle KA. Cardiac tamponade complicating proximal aortic dissection. Is pericardiocentesis harmful? Circulation 1994;90:2375-8.

3. Shimizu H, Yoshino H, Udagawa H, Watanuki A, Yano K, Ide H, et al.
Prognosis of aortic intramural hemorrhage compared with classic aortic dissection. Am J Cardiol 2000;85:792-5, A10.

4. Kaji S, Nishigami K, Akasaka T, Hozumi T, Takagi T, Kawamoto T, et al. Prediction of progression or regression of type A aortic intramural hematoma by computed tomography. Circulation 1999;100(19 Suppl):II281-6.

5. Coady MA, Rizzo JA, Hammond GL, Pierce JG, Kopf GS, Elefteriades JA. Penetrating ulcer of the thoracic aorta: what is it? How do we recognize it? How do we manage it? J Vasc Surg 1998;27:1006-15.

6. Evangelista A, Mukherjee D, Mehta RH, O'Gara PT, Fattori R, Cooper JV, et al. Acute intramural hematoma of the aorta: a mystery in evolution. Circulation 2005;111:1063-70.

7. Nienaber CA, Richartz BM, Rehders T, Ince H, Petzsch M. Aortic intramural haematoma: natural history and predictive factors for complications. Heart 2004;90:372-4.

8. Song JK, Yim JH, Ahn JM, Kim DH, Kang JW, Lee TY, et al. Outcomes of patients with acute type a aortic intramural hematoma. Circulation 2009; 120:2046-52.

9. Stanson AW, Kazmier FJ, Hollier LH, Edwards WD, Pairolero PC, Sheedy PF, et al. Penetrating atherosclerotic ulcers of the thoracic aorta: natural history and clinicopathologic correlations. Ann Vasc Surg 1986;1:15-23.

10. Erbel R, Alfonso F, Boileau C, Dirsch O, Eber B, Haverich A, et al. Diagnosis and management of aortic dissection. Eur Heart J 2001;22:1642-81.

11. Eggebrecht H, Baumgart D, Schmermund A, Herold U, Hunold P, Jakob $\mathrm{H}$, et al. Penetrating atherosclerotic ulcer of the aorta: treatment by endovascular stent-graft placement. Curr Opin Cardiol 2003;18:431-5. 